Pain - Thursday, 14 June, 16.00-17.30/Club A-B

\section{SP0128 PAIN - GENERAL ASPECTS, REVIEW ON PAIN CONTROL METHODS}

J Szechinski. Rheumatology, Medical University of Wroclaw, Poland, Wroclaw, Poland

10.1136/annrheumdis-2001.64

Pain is pervasive complaint of patients with musculoskeletal disease. There is a tremendous variability in the perception of pain that is modulated by cognitive, emotional and environmental factors. This symptom may result from both and peripheral factors and, in the individual patient, may have individual causes. Because of the interplay of this factors, treatment can entail a variety of pharmacological agents, with drugs modulating central pain as a common component even in patients with structural and inflammatory disease. In older patients and in patients with chronic inflammatory disease, frequent reevaluation may be needed to stage disease and detect intercurrent problems leading to pain. Psychosocial Assesmento of Pain in Patients Having Rheumatic Diseases as well as the Role of Physical Therapy and Physical Modalities In Pain Management is discussed.

\section{SP0129 PHARMACEUTICAL PAIN CONTROL IN RHEUMATOID ARTHRITIS}

K Viktil. Hospital Pharmacy, Diakonhjemmet Hospital, Oslo, Norway

10.1136/annrheumdis-2001.65

Relief of pain is a problem which is difficult to handle in rheumatic diseases and multidisciplinary approach is necessary. Drugs play an important role in the control of pain. There are different groups of drugs which are relevant:

- Analgesics. In general - work fast, short duration of relief. However long-lasting formulations are available. 1.1. Nonopioid analgesics - Weak to medium effect. Often as a supplement to stronger analgesics. Give no addiction. 1.2. Opioid analgesics - Strong effect. May give addiction, important to have in mind when treating pain in chronic diseases.

- Non steroidal antiinflammatory drugs/coxibs. Reduce both pain and stiffness, tenderness and swollen joints. However, interaction problems with other drugs, may affect renal function, platelet function and and give gastrointestinal (GI) problems. The new class coxibs have however reduced the GIand the platelet-problems.

- Psychotropic drugs - e.g. antidepressants, antipsychotics, antiepileptica, anxiolytica. Useful among others in neurogenic pain.

Strategies of pharmacological treatment of pain: Acute (e.g. postoperative pain) and chronic pain need different strategies.

\section{SP0130 NON-PHARMACEUTICAL PAIN CONTROL}

M Mikkelsson. Rehabilitation, The Rheumatism Foundation Hospital, Heinola, Finland

10.1136/annrheumdis-2001.66
The lecture will focus on non-pharmaceutical pain control methods in arthritis. In clinics, several pain control methods are available, although there is lack of good randomised controlled trials on different pain control methods. The results of the most interesting studies are summarised.

Acute pain is treated partly with different methods than chronic pain. Pain is always subjective. Thus, subjective report about pain is important when evaluating the effectiveness of a pain control method.

Important pain control methods are those, which patients can use themselves. These are local cold and heat, relaxation, exercise therapy, collars and splints, and some psychological methods (e.g. autohypnosis). The benefit of these methods is that they are easy available. However, they are dependent on patient's motivation.

Health professionals are needed for other pain control methods. Such methods are psychological methods (e.g. cognitive behavioural therapy and hypnosis), education, several physical therapy modalities, exercise therapies and manual physiotherapy, and acupuncture.

Pain is often a complex symptom. Usually, several treatment methods, including both pharmaceutical and non-pharmaceutical methods, are needed for achieving a good result.

\section{Psychological aspects of patients with arthritis - Saturday 16 June, 10.00-11.45/ Club A-B}

\section{SP0136 SUBJECTIVE WELL-BEING IN RHEUMATIC DISEASES - COPING OR PERSONALITY?}

L Persson. Department of Nursing, University of Göteborg, Göteborg, Sweden

\subsection{6/annrheumdis-2001.67}

Early studies dealing with the relations between psychological factors and rheumatic diseases, particularly rheumatoid arthritis, focused on whether there were some maladaptive psychological factors that could influence the onset of the disease. Personality styles like hostility ${ }^{1}$ or neurotic behavior ${ }^{2}$ were suggested. However, this line of research was soon abandoned, simply because no consistent conclusions could be made. Instead, questions were raised about the psychological and social consequences of rheumatic diseases. It was also proposed that treatment and rehabilitation should be evaluated from the patients' point of view, thus according to the effects it had on perceived symptoms, everyday functioning, well-being and social integration. Several instruments were also developed to catch various aspects of the subjective impact of the disease, for example the wellknown HAQ. ${ }^{3}$ Since then the subjective burden of having rheumatic diseases have been extensively studied. Although some studies have found that the patients as a group show small tendencies toward increased emotional distress, it is apparent that the majority of patients do not seem to differ from that of a normal population. ${ }^{4}$ In that respect rheumatic patients also resemble several other patient groups with chronic diseases. ${ }^{5}$ One major finding was also that indicators of severity of disease had surprisingly small relations with psychological health. ${ }^{6}$ Such findings led in turn to an interest for the presence of psychological factors that could "buffer" the emotional impact of the disease among the patients. Constructs that were in special focus were 